\title{
ON A FAIR FARE RATING ON A BUS LINE
}

Suppose we are given a long bus line with a single tariff. Some passengers travel along long distances while another ones along short trips. Such a single tariff is advantageous for the first mentioned passengers. However, a single tariff is inconvenient for short trip passengers. Babel, Keller, Hamacher and Schobel tried to solve this problem by dividing serviced area into zones in [1], [2] and [3]. Kohani presented similar attitude in [4]. Another way how to improve fairness is to introduce a double tariff - fare $x$ for passengers traveling at most along $k$ laps and fare $y$ for passengers traveling along more than $k$ laps. This paper shows how to determine optimal values $k, x$ and $y$.

Keywords: Tariff system, single tariff, fair double tariff, constrained extreme problem.

\section{Introduction}

Suppose we are given a bus line $L$ with $n$ bus stops. Lap of a line $L$ is a segment of a bus line between two successive bus stops. Suppose, we are given a line $L$ and the following input data

$R_{i}$ - the number of passengers traveling exactly along $i$ laps of the line $L$

$t_{i}$ - ideal, but from some reasons infeasible fair distance tariff for passengers traveling along exactly $i$ laps of the line $L$

Let $x$ and $y$ be unknown variables with the following meaning: $x$ - double tariff fare for passengers traveling along at most $k$ laps

$y$ - double tariff fare for passengers traveling along more than $k$ laps

Total fare on line $L$ in the case of ideal fair tariff is

$$
F=\sum_{i=1}^{n-1} t_{i} R_{i}
$$

Total fare on line $L$ in the case of double tariff is

$$
F_{d}=\sum_{i=1}^{k} x R_{i}+\sum_{j=k+1}^{n-1} y R_{j}
$$

Bus provider wants to keep the income from double tariff the same as the one from ideal fair tariff, i.e.:

$$
F=F_{d}
$$

$$
\sum_{i=1}^{n-1} t_{i} R_{i}=\sum_{i=1}^{k} x R_{i}+\sum_{j=k+1}^{n-1} y R_{j}
$$

\section{Measure of unfairness}

There are several ways how to express unfairness $u_{i}$ of a passenger traveling along exactly $i$ laps. A comprehensive survey of attitudes to fairness is presented in [2]. I decided to use the second power of the difference between the ideal fare and double tariff fare of considered passenger:

$$
u_{i}=\left\{\begin{array}{lll}
\left(x-t_{i}\right)^{2} & \text { if } \quad \mathrm{i} \leq \mathrm{k} \\
\left(y-t_{i}\right)^{2} & \text { if } \quad i>k
\end{array}\right.
$$

Total unfairness of all passengers on the considered line can be calculated as follows:

$$
\begin{aligned}
& U(x, y)=\sum_{i=1}^{n-1} u_{i}=\sum_{i=1}^{k} u_{i}+\sum_{j=k+1}^{n-1} u_{j}=\sum_{i=1}^{k} R_{i}\left(x-t_{i}\right)^{2}+ \\
& +\sum_{j=k+1}^{n-1} R_{j}\left(y-t_{j}\right)^{2}
\end{aligned}
$$

The sum of squared deviations is often used as a measure of unevenness. Here are several examples and reasons to accept $U(x, y)$ as a measure of unfairness by analogy:

- Let us have a discrete random value $X$ acquiring values $x_{1}, x_{2}$, ..., $x_{n}$ with probabilities $p_{1}, p_{2}, \ldots, p_{n}$ and with the mean $\bar{X}=$ $=\sum_{(\mathrm{i}=1)}^{n} x_{i} p_{i}$. In probability theory and statistics, the variance of $X$ is defined as $\operatorname{Var}(X)=\sum_{(\mathrm{i}=1)}^{n}\left(x_{i}-\bar{X}\right)^{2}-\mathrm{i}$. e. as the sum

\footnotetext{
* Stanislav Paluch

Department of Mathematical Methods, Faculty of Management Science and Informatics, University of Zilina, Slovakia,

E-mail: Stanislav.Paluch@fri.uniza.sk
} 
of squared deviations from its own mean. The variance is a measure of how far a set of numbers is spread out. It describes how far the numbers lie from the mean.

- In statistics, linear regression is an approach to modeling the relationship between a scalar dependent variable $Y$ and a explanatory variable $X$. Let us have a set of ordered pairs of real numbers $\left\{\left(x_{1}, y_{1}\right),\left(x_{2}, y_{2}\right), \ldots,\left(x_{n}, y_{n}\right)\right\}$. We want to approximate $Y$ by a linear function of $X$ :

$$
Y=f(X)=A \cdot X+B .
$$

Parameters A,B can be determined by minimization of the following sum of squared deviations

$$
\sum_{i=1}^{n}\left(y_{i}-f\left(x_{i}\right)\right)^{2}=\sum_{i=1}^{n}\left(y_{i}-A x_{i}-B\right)^{2} .
$$

In this case the sum of squared deviations of real values $y_{i}$ from theoretical values $f\left(x_{i}\right)$ is considered as a criterion of approximation quality.

- Perception of unfairness is not linear. People are willing to neglect small injustice but are sensitive to larger discrepancies. The simplest model of nonlinear perception of unfairness is a quadratic function.

- Mathematical model with objective function $U(x, y)$ allows exact analytical solution based on well known mathematical methods.

\section{Mathematical formulation and solution}

Suppose we are given a bus line $L$ with $n$ bus stops, the number $R_{i}$ of passengers traveling exactly along $i$ laps of the line $L$ and ideal fair fare $t_{i}$ for passengers traveling exactly along $i$ laps of the line $L$, both $R_{i}$ and $t_{i}$ for all $i=1,2, \ldots, n-1$.

Suppose first that we are given a fixed number $k, 1 \leq k<$ $<n-1$.

Our problem is to find a fare $x$ for passengers traveling at most $k$ laps and a fare $y$ for passengers traveling more than $k$ laps in order to minimize unfairness and to retain total income on the considered line. This leads to the following mathematical problem

\section{Minimize}

$$
\begin{aligned}
& U(x, y)=U(x, y, k)=\sum_{i=1}^{k} R_{i}\left(x-t_{i}\right)^{2}+ \\
& +\sum_{j=k+1}^{n-1} R_{j}\left(y-t_{j}\right)^{2}
\end{aligned}
$$

Subject to

$$
\sum_{i=1}^{k} x R_{i}+\sum_{j=k+1}^{n-1} y R_{j}=\sum_{i=1}^{n-1} t_{i} R_{i}
$$

This formulation is a constrained extreme problem solvable by Lagrange Multiplier Method.

Denote

$$
\begin{aligned}
& F(x, y, \lambda)=U(x, y)- \\
& -\lambda\left[\sum_{i=1}^{k} x R_{i}+\sum_{j=k+1}^{n-1} y R_{j}-\sum_{i=1}^{n-1} t_{i} R_{i}\right] \\
& F(x, y, \lambda)=\sum_{i=1}^{k} R_{i}\left(x-t_{i}\right)^{2}+\sum_{j=k+1}^{n-1} R_{j}\left(y-t_{j}\right)^{2}- \\
& -\lambda\left[\sum_{i=1}^{k} x R_{i}+\sum_{j=k+1}^{n-1} y R_{j}-\sum_{i=1}^{n-1} t_{i} R_{i}\right]
\end{aligned}
$$

Formula (1) for $U(x, y)$ defines a differentiable function on $R^{2}$ (where $R$ is the set of all real numbers). The Lagrange Multiplier Theorem asserts that if $U(x, y)$ achieves a minimum on $\mathrm{R} 2$ subject to (2), then the minimum is necessarily achieved at a point where $\frac{\partial F(x, y, \lambda)}{\partial x}=0, \frac{\partial F(x, y, \lambda)}{\partial y}=0$ and $\frac{\partial F(x, y, \lambda)}{\partial \gamma}=0$.

Let us see where Lagrange Multiplier Method tells us to look for optimal solution. It holds:

$$
\begin{aligned}
& \frac{\partial F(x, y, \lambda)}{\partial x}=2 \sum_{i=1}^{k} R_{i}\left(x-t_{i}\right)^{2}-\lambda \sum_{i=1}^{k} R_{i}= \\
& =2 x \sum_{i=1}^{k} R_{i}-2 \sum_{i=1}^{k} R_{i} t_{i}-\lambda \sum_{i=1}^{k} R_{i}=0 \\
& \frac{\partial F(x, y, \lambda)}{\partial y}=2 \sum_{j=k+1}^{n-1} R_{j}\left(y-t_{j}\right)-\lambda \sum_{j=k+1}^{n-1} R_{j}= \\
& =2 y \sum_{j=k+1}^{n-1} R_{i}-2 \sum_{j=k+1}^{n-1} R_{j} t_{j}-\lambda \sum_{j=k+1}^{n-1} R_{i}=0 \\
& \frac{\partial F(x, y, \lambda)}{\partial \lambda}=x \sum_{i=1}^{k} R_{i}+y \sum_{j=k+1}^{n-1} R_{j}-\sum_{i=1}^{n-1} t_{i} R_{i}=0
\end{aligned}
$$

It follows from (3) and (4)

$$
\begin{gathered}
x=\frac{2 \sum_{i=1}^{k} t_{i} R_{i}+\lambda \sum_{i=1}^{k} R_{i}}{2 \sum_{i=1}^{k} R_{i}}=\frac{\sum_{i=1}^{k} t_{i} R_{i}}{\sum_{i=1}^{k} R_{i}}+\frac{\lambda}{2} \\
y=\frac{2 \sum_{j=k+1}^{n-1} t_{j} R_{j}+\lambda \sum_{j=k+1}^{n-1} R_{j}}{2 \sum_{j=k+1}^{n-1} R_{j}}=\frac{\sum_{j=k+1}^{n-1} t_{j} R_{j}}{\sum_{j=k+1}^{n-1} R_{j}}+\frac{\lambda}{2}
\end{gathered}
$$

We obtain by substitution for $x$ from (6) and for $y$ from (7) into (5) 


$$
\begin{aligned}
& \left(\frac{\sum_{i=1}^{k} t_{i} R_{i}}{\sum_{i=1}^{k} R_{i}}+\frac{\lambda}{2}\right) \cdot \sum_{i=1}^{k} R_{i}+\left(\frac{\sum_{j=k+1}^{n-1} t_{j} R_{j}}{\sum_{j=k+1}^{n-1} R_{j}}+\frac{\lambda}{2}\right) \cdot \sum_{j=k+1}^{n-1} R_{j}= \\
& =\sum_{i=1}^{n-1} t_{i} R_{i}
\end{aligned}
$$

The last equation can be stepwise simplified as follows:

$$
\begin{aligned}
& \sum_{i=1}^{k} t_{i} R_{i}+\frac{\lambda}{2} \sum_{i=1}^{k} R_{i}+\sum_{j=k+1}^{n-1} t_{j} R_{j}+\frac{\lambda}{2} \sum_{j=k+1}^{n-1} R_{j}=\sum_{i=1}^{n-1} t_{i} R_{i} \\
& \left(\sum_{i=1}^{k} t_{i} R_{i}+\sum_{j=k+1}^{n-1} t_{j} R_{j}\right)+\left(\frac{\lambda}{2} \sum_{i=1}^{k} R_{i}+\frac{\lambda}{2} \sum_{j=k+1}^{n-1} R_{j}\right)=\sum_{i=1}^{n-1} t_{i} R_{i} \\
& \sum_{i=1}^{n-1} t_{i} R_{i}+\frac{\lambda}{2} \sum_{i=1}^{n-1} R_{i}=\sum_{i=1}^{n-1} t_{i} R_{i} \\
& \frac{\lambda}{2} \sum_{i=1}^{n-1} R_{i}=0 \\
& \lambda=0
\end{aligned}
$$

Substitution 0 for $\lambda$ into (6) and (7) gives

$$
x=\frac{\sum_{i=1}^{k} t_{i} R_{i}}{\sum_{i=1}^{k} R_{i}}, \quad y=\frac{\sum_{j=k+1}^{n-1} t_{j} R_{j}}{\sum_{j=k+1}^{n-1} R_{j}} .
$$

To guarantee that the function $U(x, y)$ achieves minimum at point (8) it is necessary to show that following second partial derivatives are greater than zero.

Indeed, it holds:

$$
\frac{\partial^{2} F(x, y, \lambda)}{\partial x^{2}}=2 \sum_{i=1}^{k} R_{i}>0,
$$

$$
\frac{\partial^{2} F(x, y, \lambda)}{\partial y^{2}}=2 \sum_{i=k+1}^{n-1} R_{i}>0
$$

Let us define for $k=1,2, \ldots, n-1$

$$
U^{*}(k)=\sum_{i=1}^{k} R_{i}\left(\frac{\sum_{i=1}^{k} t_{i} R_{i}}{\sum_{i=1}^{k} R_{i}}-t_{i}\right)^{2}+\sum_{j=k+1}^{n-1} R_{j}\left(\frac{\sum_{j=k+1}^{n-1} t_{j} R_{j}}{\sum_{j=k+1}^{n-1} R_{j}}-t_{j}^{2}\right.
$$

$U^{*}(k)$ is the least possible unfairness provided that double tariff uses one fare for passengers traveling less than or equal to $k$ laps and another fare for the ones traveling more than $k$ laps.

The optimum $k$ can be found by searching the set

$$
\left\{U^{*}(1), U^{*}(2), \ldots, U^{*}(n-1)\right\}
$$

for minimum. Therefore the optimum $k$ is

$$
k=\operatorname{argmin}\left\{U^{*}(1), \mathrm{U}^{*}(2), \ldots, \mathrm{U}^{*}(n-1)\right\}
$$

Corresponding optimum fares $x, y$ are determined by equations (8).

Just described procedure can be illustrated on the following example:

We can see that the unfairness $U^{*}(k)$ achieves a minimum at $k=8$ and corresponding short and long distance fares are $x=55$ and $y=114$.

\section{Conclusion}

The formulas (8) which define the fair fares for short distance and long distance passengers say that:

1. Optimum short distance fare can be computed as the total ideal fare of all short distance passengers divided by the number of all short distance passengers

2. Optimum long distance fare can be computed as the total ideal fare of all long distance passengers divided by the number of all long distance passengers

Input data for an example instance

\begin{tabular}{|l|c|c|c|c|c|c|c|c|c|c|c|c|c|c|}
\hline$i$ & 1 & 2 & 3 & 4 & 5 & 6 & 7 & 8 & 9 & 10 & 11 & 12 & 13 & 14 \\
\hline$R_{i}$ - number of passengers travelling exactly $i$ laps & 2 & 3 & 8 & 12 & 14 & 16 & 14 & 15 & 10 & 8 & 7 & 5 & 10 & 7 \\
\hline Number of passengers travelling at most $i$ laps & 2 & 5 & 13 & 25 & 39 & 55 & 69 & 84 & 94 & 102 & 109 & 114 & 124 & 131 \\
\hline$t_{i}$ - ideal fare for $i$ laps & 10 & 20 & 30 & 40 & 50 & 60 & 70 & 80 & 90 & 100 & 110 & 120 & 130 & 140 \\
\hline
\end{tabular}

Number of all passengers on the considered line equals to 131, total ideal fare is $F=\sum_{i=1}^{n-1} t_{i} R_{i}=9990$, single fare equals to $9990 / 131=76,26$. 


\section{COMMNICOIIONS}

Dependence of fair short distance fare $x$, fair long distance fare $y$ and unfairness $U^{*}(k) / 1000$ on the short distance length $k$

\begin{tabular}{|l|c|c|c|c|c|c|c|c|c|c|c|c|c|c|}
\hline$k$ - number of laps for short distance fare & 1 & 2 & 3 & 4 & 5 & 6 & 7 & $\mathbf{8}$ & 9 & 10 & 11 & 12 & 13 & 14 \\
\hline Short distance fare $x$ (rounded) & 10 & 16 & 25 & 32 & 38 & 45 & 50 & $\mathbf{5 5}$ & 59 & 62 & 65 & 68 & 73 & 76 \\
\hline Long distance fare $y$ (rounded) & 77 & 79 & 82 & 87 & 92 & 99 & 106 & $\mathbf{1 1 4}$ & 120 & 126 & 131 & 134 & 140 & - \\
\hline Unfairness $U^{*}(k) / 1000$ & 138 & 128 & 109 & 87 & 68 & 53 & 45 & $\mathbf{4 4}$ & 47 & 55 & 68 & 82 & 117 & 147 \\
\hline
\end{tabular}

Another interesting discovery of just presented optimization procedure is that $\lambda=0$. This fact implies that the minimization of $U(x, y)(1)$ without constraint (2) leads to the same result as the one of the constrained extreme problem (1), (2).

Future work will be focused to assessment of value $R_{i}$ - the number of passengers traveling exactly along $i$ laps as a function of $i$ and to more precise specification of ideal fare $t_{i}$. Another gene-

ralization of this method can be obtained by considering non equal laps. Most difficult problem can arise if we will suppose that $R_{i}$ is a function not only of $i$ but also of corresponding fare.

\section{Acknowledgement}

This work was supported by the research grant APVV-076011 "Designing of Fair Service Systems on Transportation Networks".

\section{References}

[1] BABEL, L., KELLER, H.: Design of Tariff Zones in Public Transportation Networks: Theoretical Results and Heuristics, Mathematical Methods of Operations Research 58, 2003, Springer-Verlag, pp. 359-374.

[2] HAMACHER, H., W., SCHOBEL, A.: Design of Zone Tariff Systems in Public Transportation, Operation Research, vol. 52, No. 6 , November-December 2004, pp. 897-908, ISSN 0030-364X.

[3] SCHOBEL, A.: Zone Planning in Public Transportation. L. Bianco, P. Toth, eds. Advanced Methods in Transportation Analysis. Springer Verlag, Berlin, 1996, pp. 117-134.

[4] KOHANI, M.: Exact Approach to the Tariff Zones Design Problem in Public, Proc. Of the $30^{\text {th }}$ intern. conference: Mathematical Methods in Economics, Karvina, September 2012, Silesian University in Opava, School of Bussines Administration, 2012, pp. 426-431.

[5] BERTSIMAS, D., FARIAS, V. F., TRICHAKIS, N.: The Price of Fairness. Operations Research, 59(1), pp. 17-31, 2011. 